Check for updates

Cite this: J. Mater. Chem. A, 2021, 9, 21845

Received 26th July 2021

Accepted 31st August 2021

DOI: $10.1039 / \mathrm{d} 1 \mathrm{ta0} 290 \mathrm{e}$

rsc.li/materials-a

\title{
Evolution of ferroelectric domains in methylammonium lead iodide and correlation with the performance of perovskite solar cells $\uparrow$
}

\author{
Tobias Leonhard, ${ }^{\mathrm{ab}}$ Holger Röhm, (D) ${ }^{\mathrm{ab}}$ Fabian J. Altermann, (D) ${ }^{\mathrm{c}}$ \\ Michael J. Hoffmann (iD ac and Alexander Colsmann (iD *ab
}

\begin{abstract}
While more and more experimental evidence for the ferroelectricity of methylammonium lead iodide $\left(\mathrm{MAPb}_{3}\right)$ is being reported in the literature, the scientific community still controversially discusses whether or not the ferroelectric polarization has any influence on the performance of perovskite solar cells. In this work, the evolution of ferroelectric domains and their polarization orientation in $\mathrm{MAPbl}_{3}$ thin films during thermal annealing are investigated using piezoresponse force microscopy (PFM) and Kelvin probe force microscopy (KPFM). Right after deposition and annealing for $5 \mathrm{~s}$, small grains with nonuniform crystal orientation and polarization are formed. During the next 30-60 s, these small grains increase in diameter and large ferroelectric domains with out-of-plane polarization appear. In the annealing regime of several minutes to one hour, these large grains produce uniform domains with alternating in-plane polarization and (110) texture. The corresponding $\mathrm{MAPbl}_{3}$ solar cells show a distinct performance enhancement and improved operational stability if the ferroelectric polarization is oriented in-plane. In contrast, solar cells with out-of-plane-polarized $\mathrm{MAPbl}_{3}$ exhibit only moderate fill factors and reduced open-circuit voltages.
\end{abstract}

\section{Introduction}

Organic metal halide perovskites $(\mathrm{OMH})$ are one of the most exciting material classes of the last decade which find their application in perovskite solar cells, light-emitting diodes and other optoelectronic applications. The remarkable photovoltaic performance of polycrystalline OMH thin-films has its origin in efficient charge carrier transport and low recombination losses. ${ }^{1-3}$ In order to unravel the fundamental mechanisms behind these exceptional charge carrier dynamics, several computational studies investigated the crystal properties of the archetypical methylammonium lead iodide $\left(\mathrm{CH}_{3} \mathrm{NH}_{3} \mathrm{PbI}_{3}, \mathrm{MAPbI}_{3}\right)$ and suggested that $\mathrm{MAPbI}_{3}$ may be ferroelectric. ${ }^{4-6}$ By definition, ferroelectric materials exhibit a spontaneous polarization, which can be reversed or aligned under an external electric field. ${ }^{7,8}$ This spontaneous and adjustable polarization triggers the formation of polar domains with alternating polarization. ${ }^{9}$ According to simulations, the microscopic electrical fields

${ }^{a}$ Karlsruhe Institute of Technology, Material Research Center for Energy Systems (MZE), Strasse am Forum 7, 76131 Karlsruhe, Germany. E-mail: alexander. colsmann@kit.edu

${ }^{b}$ Karlsruhe Institute of Technology, Light Technology Institute (LTI), Engesserstrasse 13, 76131 Karlsruhe, Germany

${ }^{c}$ Karlsruhe Institute of Technology, Institute for Applied Materials - Ceramic Materials and Technologies (IAM), Haid-und-Neu-Strasse 7, 76131 Karlsruhe, Germany

$\dagger$ Electronic supplementary information (ESI) available. See DOI: $10.1039 / \mathrm{d} 1 \mathrm{ta} 06290 \mathrm{e}$ within these domains can help the efficient charge carrier separation..$^{\mathbf{1 0}-\mathbf{1 2}}$ At the same time, channel-like pathways are created in the crystal, which transport the photo-generated charge carriers towards the respective electrodes along safe pathways.

On the experimental side, in early works, $\mathrm{MAPbI}_{3}$ thin-films were investigated for ferroelectricity mostly by resonanceenhanced piezoresponse force microscopy (PFM), consistently showing striped domain patterns with alternating piezoresponse. ${ }^{13,14}$ The interpretation of this alternating piezoresponse, however, fueled a vivid debate on the ferroic properties of $\mathrm{MAPbI}_{3}$. While the alternating piezoresponse of domain assemblies generally is indicative of ferroelectricity, it was rightfully pointed out that the resonance enhancement during PFM measurements may induce measurement artefacts. ${ }^{15-17}$ Also, mobile ionic charges may populate the surface of the $\mathrm{MAPbI}_{3}$ layer and corrupt the measurement data. ${ }^{18}$ While these are valid concerns, more recently, other observations and material properties were reported and correlated with PFM data in favor of ferroelectricity. These data include anisotropy of the PFM signal in vertical and horizontal direction, ${ }^{19}$ a correlation of the piezoresponse with the $c$-axis of the tetragonal crystal, ${ }^{19}$ a surface potential modulation of occasional out-of-plane polarized domains ${ }^{20}$ and the displacement of iodine in the tetragonal unit cell, creating an electrical dipole. ${ }^{21}$ In addition, the switching of domains and shifting of domain walls under external polarizing electrical fields $\mathrm{s}^{22}$ as well as a hysteresis loop 
at lower temperature were observed. ${ }^{23}$ Notably, $\mathrm{MAPbI}_{3}$ is a semiconductor which exhibits properties that are somewhat different from classical ferroelectric dielectrics, e.g., external polarization fields have to be rather weak to not destroy the crystal (creeping poling). ${ }^{22,24}$ The effects occurring in a ferroelectric semiconductor must not be confused with the bulkphotovoltaic effect which only occurs in dielectrics. ${ }^{25,26}$ Although controversial discussions surrounding the ferroelectric or non-ferroelectric nature of $\mathrm{MAPbI}_{3}$ continue to fill the scientific literature, ${ }^{27-33}$ the authors of this study consider the comprehensive set of experimental findings compelling in favor of ferroelectricity. So far, no other theory can explain all of the observed domain and material properties. This is why we henceforth discuss our experimental findings in light of $\mathrm{MAPbI}_{3}$ being a ferroelectric semiconductor.

The discussion surrounding the ferroelectricity of $\mathrm{MAPbI}_{3}$ is often considered purely academic as, to date, no experimental evidence was produced of the impact of the ferroelectric domains on the solar cell performance. In this work, we set out to experimentally correlate ferroelectric polarization and solar cell performance. To this end, we investigate the formation and evolution of ferroelectric domains during the fabrication of polycrystalline $\mathrm{MAPbI}_{3}$ thin-films and subsequently build solar cells from these samples. The analysis of the microstructure reveals that the duration of thermal annealing, which is a common step in nearly all manufacturing processes of $\mathrm{OMH}$ perovskite solar cells, is decisive for the domain formation and the orientation of the polarization. We attribute the observed changes of the domain structure to an interplay of mechanical stress and depolarizing fields during grain growth and thin-film formation. ${ }^{34}$ In particular, the sample cooling below the Curie temperature $T_{\mathrm{C}}\left(\mathrm{MAPbI}_{3}: T_{\mathrm{C}}=55^{\circ} \mathrm{C}\right.$ (ref. 35)) plays a critical role in this process, since the cubic-to-tetragonal phase transition leads to mechanical strain and spontaneous polarization $P_{\mathrm{S}}$. Solar cells incorporating $\mathrm{MAPbI}_{3}$ layers with different polarization orientations show distinctly different current densityvoltage $(J-V)$ characteristics.

\section{Results and discussion}

\subsection{Ferroelectric domains}

All our experiments were carried out on $\mathrm{MAPbI}_{3}$ layers with a thickness of $300 \mathrm{~nm}$ as commonly implemented as light harvesting layers in solar cells. $\mathrm{MAPbI}_{3}$ was deposited from lead iodide $\left(\mathrm{PbI}_{2}\right)$ and methylammonium iodide (MAI) in a two-step process onto a glass/indium tin oxide (ITO)/poly(3,4-ethylenedioxythiophene):polystyrenesulfonate (PEDOT:PSS) substrate as described in our previous studies. ${ }^{\mathbf{1 4 , 1 9}}$ Then the samples were thermally annealed at $100{ }^{\circ} \mathrm{C}$ under solvent vapor atmosphere that was maintained by covering each sample with a small Petri dish (volume approx. $5 \mathrm{~cm}^{3}$ ). The microstructure of grains and the domain formation were then assigned to the different annealing times. We used piezoresponse force microscopy (PFM) as a versatile characterization tool that allows probing of out-of-plane (vertical PFM; VPFM) and in-plane (lateral PFM; LPFM) piezoresponse independently. In combination with standard atomic force microscopy (AFM), PFM further lets us explore the relation between grain growth and ferroelectric domain formation. Notably, upon continuous measurements of the piezoresponse of the sample under an external electrical bias, the soft $\mathrm{MAPbI}_{3}$ layers may be damaged, allowing only a limited number of PFM measurements at each sample position. Since any long-term exposure to humid air during characterization can damage the perovskite samples, all fabrication and characterization steps were conducted in an inert nitrogen atmosphere inside a glovebox. Yet, the sample position had to be changed between different sets of measurements.

2.1.1 Evolution of the $\mathrm{MAPbI}_{3}$ microstructure during annealing. In common perovskite solar cell fabrication processes, the samples are thermally annealed to trigger conversion of the precursors $\mathrm{PbI}_{2}$ and $\mathrm{MAI}$ to $\mathrm{MAPbI}_{3}$ (ref. 36 and 37 ) as well as to produce an appropriate grain size ${ }^{38}$ and microstructure. ${ }^{39}$ Common annealing temperatures are on the order of $100{ }^{\circ} \mathrm{C},{ }^{40}$ and common annealing periods last for about $60 \mathrm{~min} .{ }^{41}$ In the following, we set out to investigate, how the annealing time affects grain growth and the microstructure of $\mathrm{MAPbI}_{3}$ at the common temperature of $100{ }^{\circ} \mathrm{C}$, which is well above its Curie temperature $T_{\mathrm{C}}=55{ }^{\circ} \mathrm{C}$.

Fig. 1, row 1 depicts a series of AFM topography images of five $\mathrm{MAPbI}_{3}$ samples that were fabricated using identical processing parameters, but different annealing times after the initial precursor-to-perovskite conversion that occurs within the first 5 s of annealing: sample $1 \rightarrow 5 \mathrm{~s}$; sample $2 \rightarrow 30 \mathrm{~s}$; sample $3 \rightarrow 60 \mathrm{~s}$; sample $4 \rightarrow 3 \mathrm{~min}$; sample $5 \rightarrow 60 \mathrm{~min}$. Throughout this series of five samples, we observed continuous grain growth towards longer annealing times, mediated by the solvent atmosphere under the Petri dish. This grain growth is most significant within the first $30 \mathrm{~s}$ of annealing. While $5 \mathrm{~s}$ of annealing formed small roundish grains with a diameter of 200-300 nm, $30 \mathrm{~s}$ and $60 \mathrm{~s}$ of annealing resulted in more flat grains with higher diameter that replaced some of the small grains with diameters below $300 \mathrm{~nm}$ (see ESI Fig. S1† for a statistical analysis). Annealing for several minutes led to coalescence of grains for diameters of several micrometers.

The annealing time does not only determine the grain size but also the crystallographic properties of the $\mathrm{MAPbI}_{3}$ grains. The X-ray diffraction (XRD) measurements on samples that were annealed for $5 \mathrm{~s}, 30 \mathrm{~s}$ and $60 \mathrm{~min}$ (equivalent to samples 1, 2 and 5 in Fig. 1) in Fig. 2 provide an integrated information of the grain orientation of the layer. The data in Fig. 2a confirms that, after five seconds, the conversion of the precursor to perovskite is complete and no notable precursor residues persist. The diffractograms also show the evolution of the orientation. After $5 \mathrm{~s}$, we find mostly non-uniformly oriented grains with some minor texture in [002] and [110] direction. After longer annealing for $30 \mathrm{~s}$ (Fig. 2b), together with an increase of grain size and crystallinity, we still observe the coexistence of (002) and (110) orientations with an enhancement of the (002) peak. After $60 \mathrm{~min}$ (Fig. 2c), the (110) peak and orientation prevail while the (002) peak and orientation have vanished, which is in accordance with the predominant (110) texture that was reported in previous work on $\mathrm{MAPbI}_{3}$ absorber layers that were optimized for solar cell integration. ${ }^{19}$ A (001) 


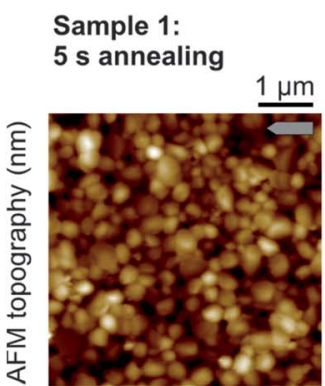

$0 \longrightarrow 100$

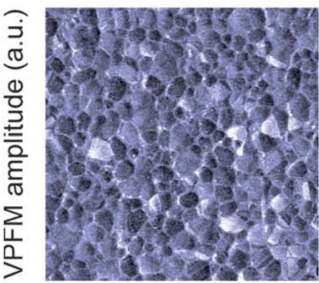

0.0
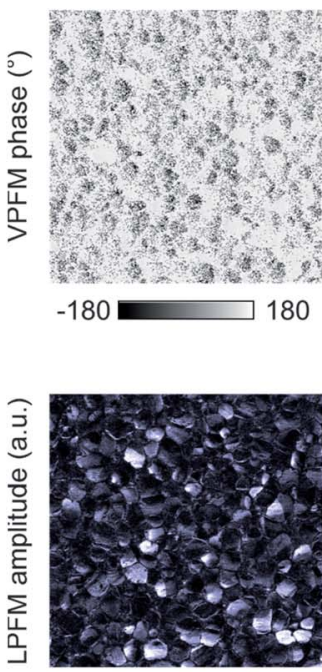

0.0

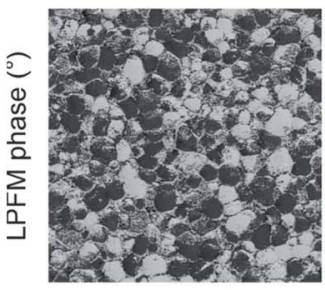

$-180 \square 180$

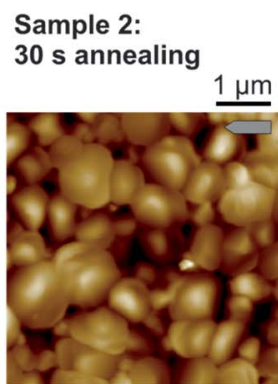

$0 \square 145$

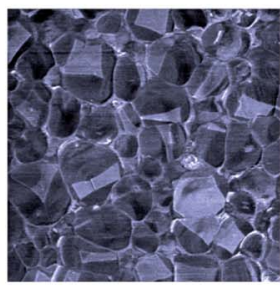

$0.0 \longleftarrow 1.0$
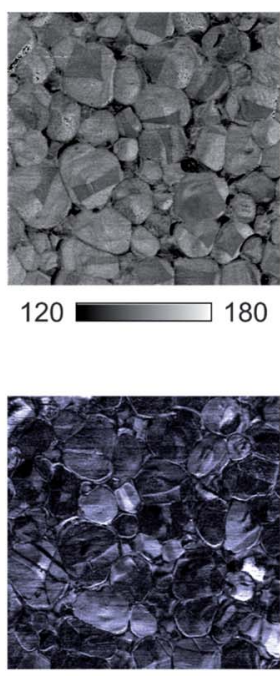

0.0

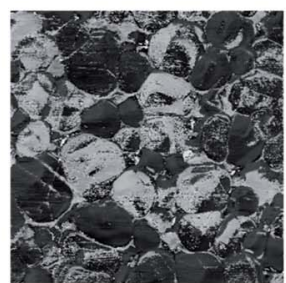

$-180$

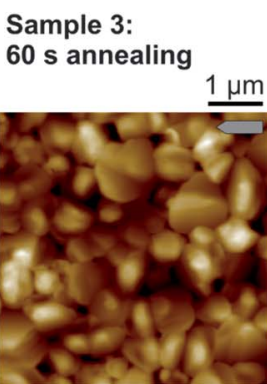

0

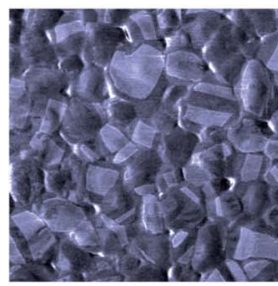

0.0

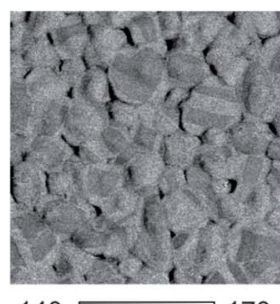

140

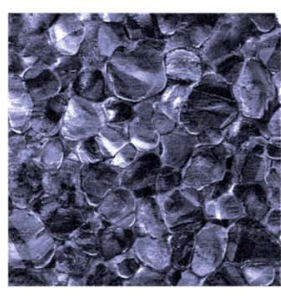

0.0

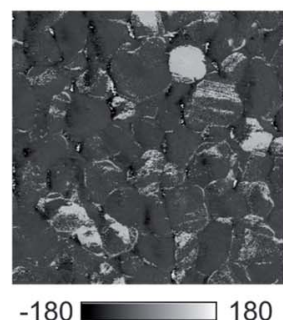

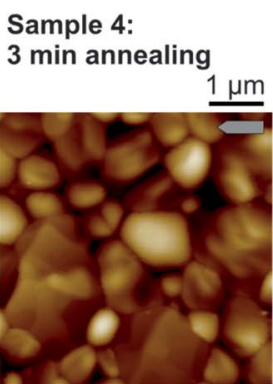

0

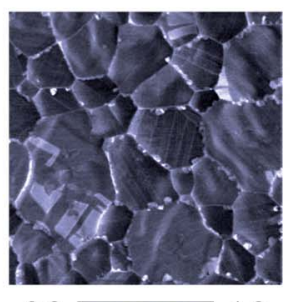

0.0
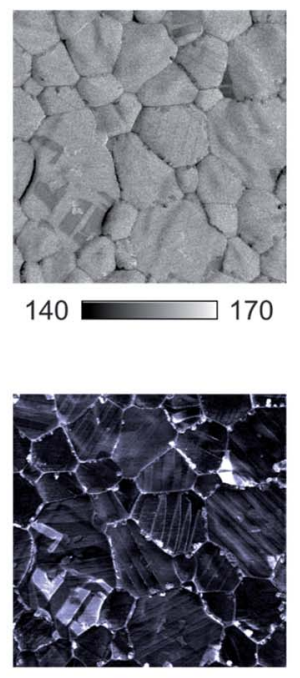

0.0

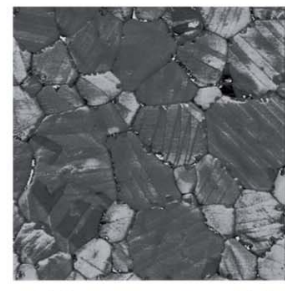

$-180$
Sample 5:

$60 \mathrm{~min}$ annealing

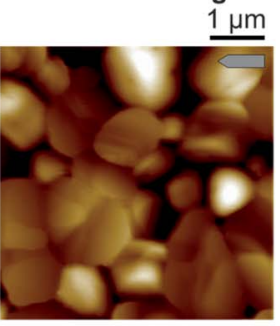

$0 \square 160$
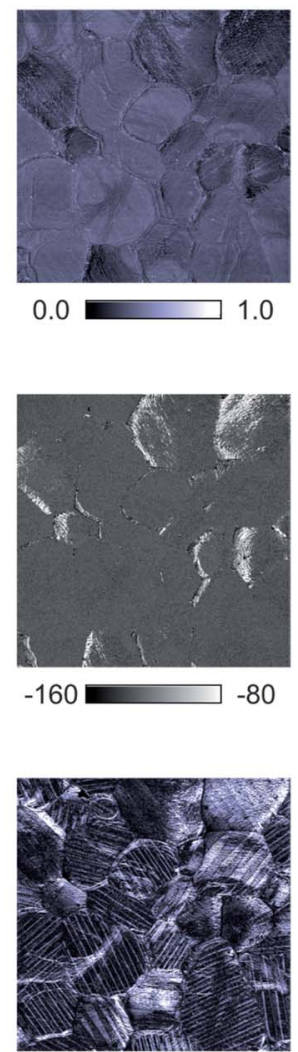

$0.0 \square 1.0$

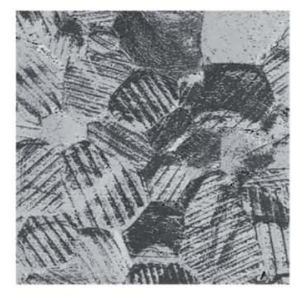

$-180 \square 180$

Fig. 1 Effects of thermal annealing on the microstructure of polycrystalline MAPbl 3 thin-films. Samples 1-5 (columns from left to right) were exposed to thermal annealing for $5 \mathrm{~s}, 30 \mathrm{~s}, 60 \mathrm{~s}, 3 \mathrm{~min}$ and $60 \mathrm{~min}$, which caused modifications to grain sizes and domain structures. The rows represent the AFM topography, VPFM amplitude, VPFM phase, LPFM amplitude, LPFM phase. The cantilever orientation is indicated by the small grey arrow. Most of the grain growth occurs during the first 30 seconds of annealing (samples 1 and 2). Due to small grain diameters and various surface facets, each grain in sample 1 comprises uniform out-of-plane and in-plane ferroelectric polarization components. Upon longer annealing and grain growth, the polarization changes and evolves towards a predominant in-plane orientation with alternating domains (sample 5). 


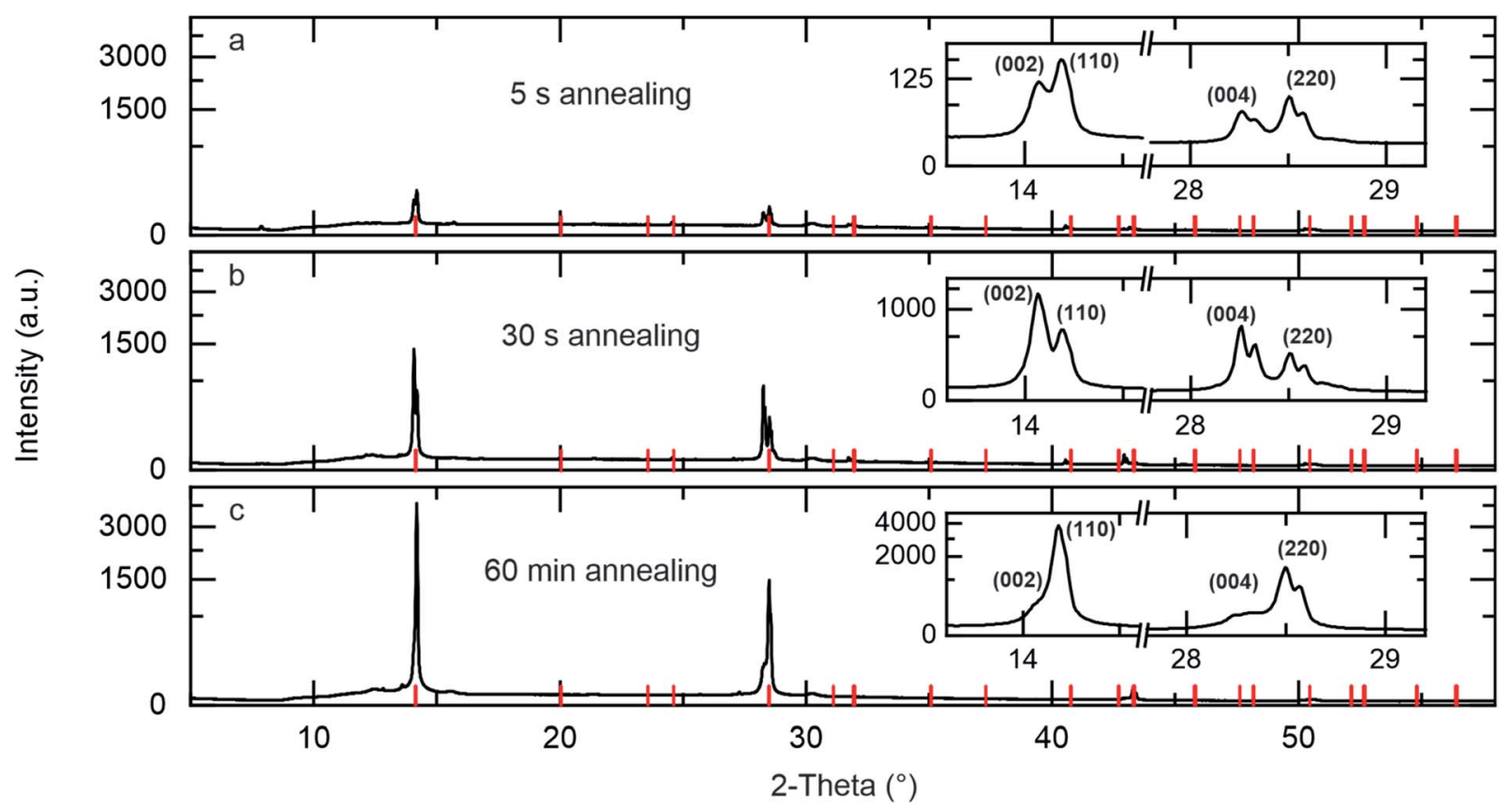

Fig. 2 XRD data of $\mathrm{MAPbl}_{3}$ samples that were thermally annealed for (a) $5 \mathrm{~s}$, (b) $30 \mathrm{~s}$ and (c) 60 min. After $5 \mathrm{~s}$ of annealing, no more precursor residues were found. The texture gradually changes from non-uniform with local (002) and (110) orientation to a prevailing (110) texture.

texture implies that the $c$-axis of the tetragonal perovskite unit cell is oriented out-of-plane, i.e., the $c$-axis is oriented perpendicular to the substrate, whereas a (110) texture corresponds to the $c$-axis being in-plane, i.e. the $c$-axis is oriented parallel to the substrate.

Since the $c$-axis correlates with the direction of ferroelectric polarization in a tetragonal perovskite unit cell, we can conclude on the dominant ferroelectric polarization orientation from the XRD data. ${ }^{19}$ Yet, a more detailed and spatially resolved microscopic picture of the ferroelectric polarization can be gained using PFM. In contrast to XRD, PFM can monitor ferroelectric domains with high spatial resolution by detecting (alternating) piezoresponse which is correlated to the crystal polarization. The two predominant orientations of the c-axis, inplane and out-of-plane, call for using lateral and vertical PFM in order to distinguish in-plane and out-of-plane polarization of the sample. Rows 2 and 3 of Fig. 1 depict the VPFM amplitudes and phases of samples 1-5. Rows 4 and 5 depict the corresponding LPFM amplitude and phase. In these PFM micrographs, every image pixel has a size of $13 \times 13 \mathrm{~nm}^{2}$ allowing a detailed observation of domains.

Strikingly, but in accordance with the observed crystal texture, we found a gradual change of the ferroelectric polarization direction towards prolonged annealing times. On sample 1 with the shortest annealing time of $5 \mathrm{~s}$, we find contrast in amplitude and phase of both VPFM and LPFM between individual small grains and their neighbors. While individual grains show uniform PFM amplitudes, which indicate a uniform polarization and orientation of each grain, the piezoresponse varies between different grains. In particular, we observed grains with both out-of-plane and in-plane polarization components, which is in accordance with the different grain orientations in sample 1 (XRD measurements, Fig. 2a). In addition, the various surface facets and surface potentials of curved grains with varying slopes may affect the PFM signal. After annealing for $30 \mathrm{~s}$ (sample 2), we observed large trapezoidal domains (bright areas in the VPFM amplitude) with an out-of-plane polarization component. On sample 3, which was annealed for $60 \mathrm{~s}$, a similar domain configuration was created. As we will discuss in Section 2.3, the areas of low VPFM amplitude represent domains of in-plane polarization. After 3 min of annealing (sample 4), we observed a notably different domain pattern: while only a few grains exhibit trapezoidal domains with high VPFM amplitude, most grains possess clear domain features in LPFM, i.e., both LPFM amplitude and phase of sample 4 show striped domains with alternating polarization. On sample 5, which was annealed for $60 \mathrm{~min}$, the VPFM contrast finally vanishes and a distinct LPFM contrast indicates an almost complete transformation to in-plane polarization direction. Both, PFM amplitude and phase, confirm striped domain patterns on each $\mathrm{MAPbI}_{3}$ grain of sample 5 that clearly differ in size and shape from the trapezoidal domains with outof-plane polarization that were visible on samples 2 and 3 . The enhanced LPFM amplitudes at domain walls of sample 5 might indicate $90^{\circ}$-domain configurations, where the polarization orientation of neighboring domains forms an angle of $90^{\circ} .{ }^{14} \mathrm{We}$ finally note that neither the vertical domains with out-of-plane piezoresponse nor the lateral domains are visible in the topography. We can also exclude any topographic crosstalk as the origin of the domain contrast since the domains often appear to continue even across grain boundaries, changes in slope and curvature of grains.

Altogether, this set of measurements reveals that the annealing time influences the size of the grains as well as the 

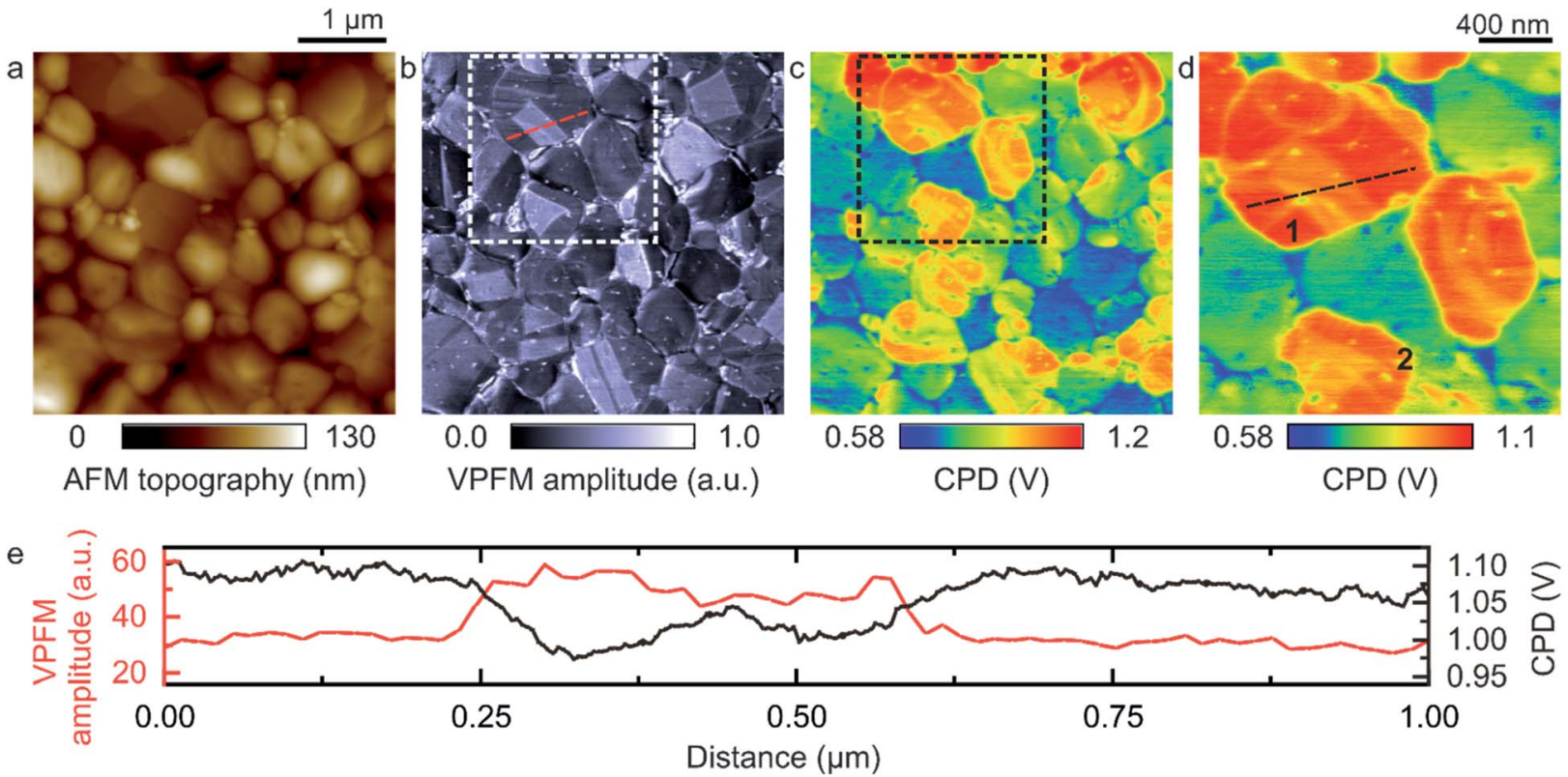

Fig. 3 Modulation of the surface potential by ferroelectric domains with out-of-plane polarization components. (a) Topography and (b) VPFM amplitude of a representative $\mathrm{MAPbl}_{3}$ layer that was annealed for $60 \mathrm{~s}$. Amidst the vast areas of low VPFM amplitude, some grains exhibit large domains that produce high VPFM amplitudes, attributable to local out-of-plane polarization components. (c) The FM-KPFM image of the same sample position shows a varying contact potential difference (CPD) between grains due to different grain orientations, but also due to a modulation of the CPD by domains with out-of-plane polarization. (d) From the enlarged sample area, the difference in CPD of about $50 \mathrm{mV}$ can be deduced by comparing out-of-plane polarized domains and their surrounding area. The lower CPD on the domains with out-of-plane polarization indicates that the polarization vector points downwards. (e) Line scans along the dashed lines in $b+d$ show differences in CPD of about $50 \mathrm{mV}$ at vertical domains with increased VPFM amplitude.

size and the polarization orientation of the ferroelectric domains. Starting with small grain sizes and a random polarization orientation containing both out-of-plane and in-plane polarization components, the grain size increases during a short period of annealing. After 30-60 s of annealing, we find significant amounts of domains with out-of-plane polarization components. Prolonging the time of annealing further does not cause notable changes to the grain diameter, but transforms the polarization to a vastly predominant in-plane orientation.

Notably, we observed the domain features also with stiffer cantilevers, which reduce electrostatic contributions, but at the same time increase the sample wear. In general, the number of PFM measurements on the same sample position is limited by sample wear, requiring occasional sample replacements. This, however, does not affect the comparability of the samples and measurement results, since the qualitative observations were reproduced several times on identical samples.

2.1.2 Vertical polarization: up- or downward? On samples 2 and 3 , grains with diameters of several hundred nanometers and large domains with homogenous piezoresponse and trapezoidal shape have formed. The strong contrast between areas of high and low amplitudes in VPFM indicates domains with out-of-plane polarization, but whether these domains exhibit up- or downward polarization, to this point, remains unclear. The polarization orientation is usually determined from the PFM phase, ${ }^{42}$ but the use of contact resonance enhancement, which is essential to improve the signal-to-noise ratio and hence to produce sufficient measurement contrast, impedes the exact determination of the polarization orientation (up- or downward polarization) ${ }^{43}$ Therefore, we took advantage of another ferroelectric domain signature: in domains with prevailing out-ofplane polarization components, surface charges accumulate at the interfaces to the bottom electrode and at the grain surface in order to compensate the electrical field from the spontaneous polarization. This charge accumulation leads to a modulation of the surface potential depending on the polarization orientation in up- or downward direction, ${ }^{44}$ which can be monitored with frequency modulated Kelvin Probe Force Microscopy (FMKPFM).

We used this measurement of the contact potential difference (CPD) to reveal surface potential differences between vertical and lateral domains with high spatial resolution. Depending on the polarization orientation of an individual vertical domain, its surface potential is either higher or lower than that of the surrounding grain surface. Fig. 3 shows another sample that was thermally annealed for $60 \mathrm{~s}$, producing similar features in topography (Fig. 3a) and VPFM amplitude (Fig. 3b) as sample 3 (Fig. 1). Fig. $3 \mathrm{c}$ and the sampling area magnification in Fig. 3d depict the CPD of the same sample position and reveal different surface potentials on the $\mathrm{MAPbI}_{3}$ grains. While many large grains possess high CPDs, most small grains possess lower CPDs, which might indicate a different grain orientation and hence different crystal facets, well agreeing to what we observed on sample 1 in Fig. $1 .^{19,45}$ On the representative grains 1 and 2 in Fig. 3d, we observe a homogenously high CPD, but the trapezoidal domains with a high VPFM amplitude 

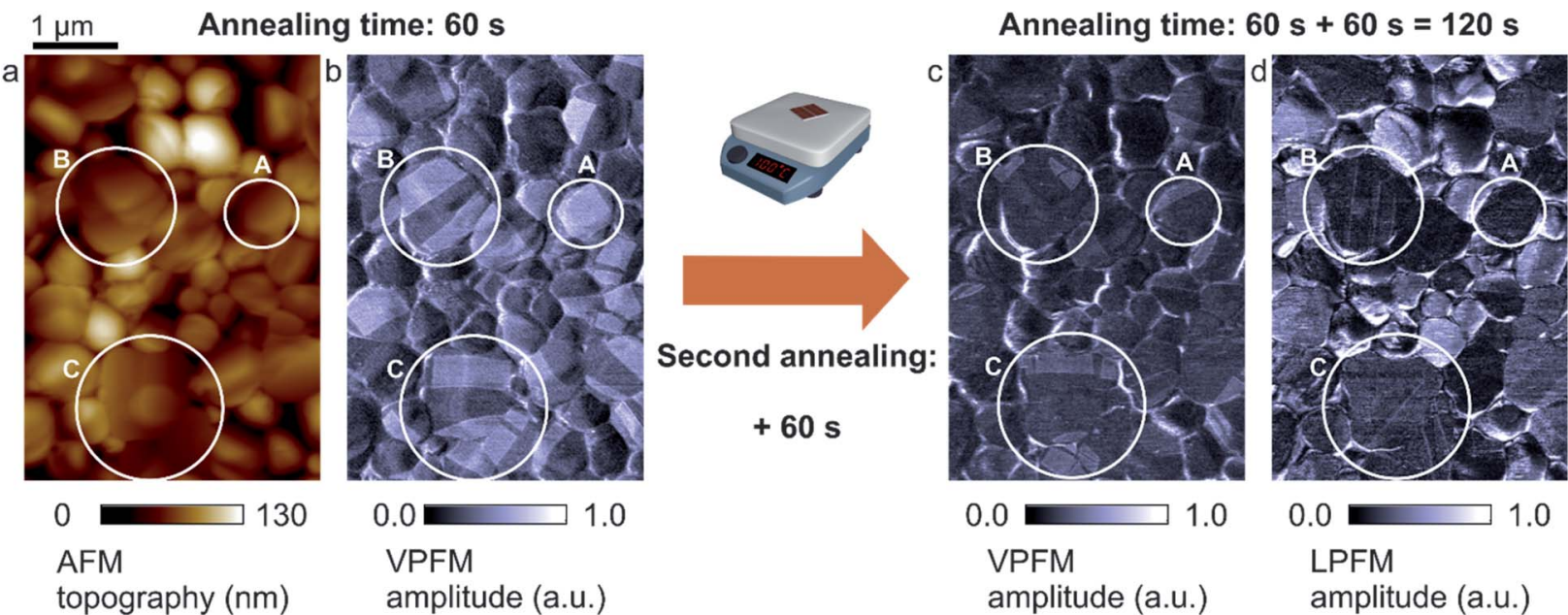

Fig. 4 Ferroelectric domains before and after a second annealing step. (a) Sample topography. (b) The VPFM amplitude of a sample after the initial $60 \mathrm{~s}$ of annealing shows out-of-plane polarized domains. (c) After the second annealing for $60 \mathrm{~s}$, the vertical domains shrink in size and partly vanish (marked in white). (d) At the same time, alternating domains with in-plane polarization occur in LPFM, displacing the vertical domains.

exhibit a reduced CPD. In Fig. 3e, the CPD line scan along the dashed line in Fig. 3d shows a local CPD reduction of about $50 \mathrm{mV}$, which correlates to the high local VPFM amplitude in Fig. 3b. In this KPFM measurement setup, a higher CPD corresponds to a higher absolute work function. Hence, we attribute this reduced CPD to an accumulation of negative surface charges, e.g. iodine ions or electrons, to compensate the polarization fields within the domains. We therefore conclude that the polarization vector points downwards (towards the substrate) in the two vertical domains of the investigated sample region. Throughout all samples, FM-KPFM measurements consistently show the same reduced CPD on all domains with high VPFM amplitude relative to other parts of the grains with low VPFM amplitude (further examples are provided in the ESI Fig. S2†).

2.1.3 From out-of-plane to in-plane polarization. The transformation from vertical (out-of-plane) to lateral (in-plane) polarization is a notable process that occurs in the time regime beyond $60 \mathrm{~s}$ of thermal annealing. In classical ferroelectric ceramics, the domain formation follows the minimization of the free energy in order to obtain the energetically most favorable state. $^{9}$ The change of domain configurations, when domains gradually split, is commonly referred to as "domain aging", ${ }^{46,47}$ which releases strain from the crystal and transforms the system towards an energetically favorable domain configuration. This formation of new domains continues until the energy, that is required to form new domain walls, equals the energy gain from reduced electrostatic and mechanical forces. ${ }^{48}$ To gain insight into this polarization transformation in $\mathrm{MAPbI}_{3}$ thin-films, we annealed another sample for $60 \mathrm{~s}$ and afterwards subdued it to a second annealing step $\left(60 \mathrm{~s}, 100^{\circ} \mathrm{C}\right.$ under a Petri dish). Fig. 4 depicts the sample topography (Fig. 4a) and VPFM response (Fig. $4 \mathrm{~b}$ ) before the second annealing, which matches the observations described in
Section 2.1, as well as VPFM (Fig. 4c) and LPFM (Fig. 4d) of the very same sample area after the second thermal annealing step.

The VPFM image in Fig. 4b highlights some domains with vertical polarization components: position A marks a grain with a relatively small diameter. Its homogenously high VPFM amplitude indicates a uniform vertical polarization throughout the grain. Positions B and C mark larger grains comprising domains of different polarization orientation, exhibiting alternating high- and low-amplitudes. After the second annealing, the shape of the grains and their topography persisted (ESI Fig. S3†), whereas the domain sizes and shapes changed significantly.

Fig. 4c shows that areas with low VPFM amplitude grow in size whereas domains with high amplitude shrink. For example, on the previously out-of-plane polarized, small grain at position A, two domains formed. Of these two domains, only the narrower domain at the top of the area exhibits high VPFM amplitude and hence vertical polarization (Fig. 4c). The larger domain exhibits only low VPFM amplitude. The domains at positions $\mathrm{B}$ and $\mathrm{C}$ seemingly migrate towards the grain boundaries, leaving behind areas of low VPFM amplitude. In these areas of low VPFM amplitude, small, needle-like domains emerge showing contrast in LPFM (Fig. 4d). Since these domains are neither visible in VPFM nor do correlate to topography features, we conclude that they possess in-plane polarization. These needle-shaped, in-plane polarized domains start to grow from the grain boundaries and spread over the grain, thus replacing domains of out-of-plane polarization with in-plane polarization (ESI Fig. S4†).

A more detailed view on the displacement of vertical domains by horizontal domains is provided in Fig. 5a-c, comparing topography, VPFM and LPFM of a typical sample that was annealed for 2 min (the corresponding VPFM and LPFM phase images can be found in the ESI Fig. S5†). In the areas of low VPFM amplitude, the LPFM amplitude shows 
lateral domains drawing through the sample. During the transformation process, the sample exhibits distinct features of both vertical and lateral domains. The area highlighted with dashed rectangles in Fig. 5a-c is magnified in Fig. $5 \mathrm{~d}-\mathrm{f}$ revealing another characteristic transformation feature: on the encircled grain, the VPFM amplitude in Fig. 5e is weak except for two domains that show the characteristic trapezoidal domain shape and distinct contrast stemming from an out-ofplane polarization. The domain wall of this vertical domain exhibits a zigzagged indentation. The origin of this indentation can be clearly identified in the LPFM image in Fig. $5 \mathrm{f}$ as a needle-shaped lateral domain. Similar to ferroelectric domains in $\mathrm{BaTiO}_{3},{ }^{49}$ this lateral domain emerges near one of the grain boundaries and extends over the grain surface until it hits a vertical domain wall. The domain frustration at the point of contact leads to an needle-shaped convergence of the lateral domain and comes along with a clear indentation of the vertical domain wall. Notably, this single grain comprises three types of domains: besides domains with out-of-plane polarization, we find domains with two different in-plane polarization directions, which is another striking evidence for the ferroelectric nature of $\mathrm{MAPbI}_{3}$. We observed similar features at other sample positions as depicted in the ESI Fig. S6. $\dagger$

2.1.4 Towards stable domain configurations with in-plane polarization. The emergence of ferroelectric domains with inplane polarization and the concomitant quenching of domains with out-of-plane polarization still do not result in a thermodynamic equilibrium state. Instead, in the annealing regime of several minutes, we observe further changes in the domain configuration towards longer annealing times, away from the needle-shaped laterally polarized domains towards a parallel domain stripe pattern. This became visible when comparing sample properties after $3 \mathrm{~min}$ of annealing and after a second annealing step of $2 \mathrm{~min}$.

Fig. 6 shows a direct comparison of the topography $(a+c)$ and LPFM phase $(b+d)$ of a representative sample before and after the second annealing step (the corresponding LPFM amplitude images are provided in ESI Fig. S7 $\dagger$ ). The LPFM

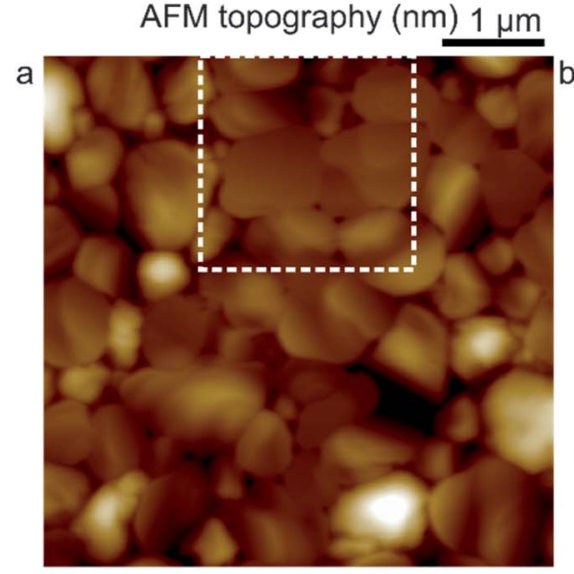

0

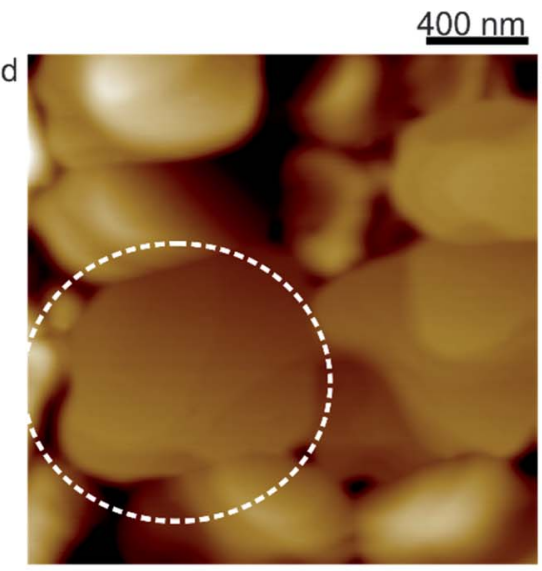

0
180

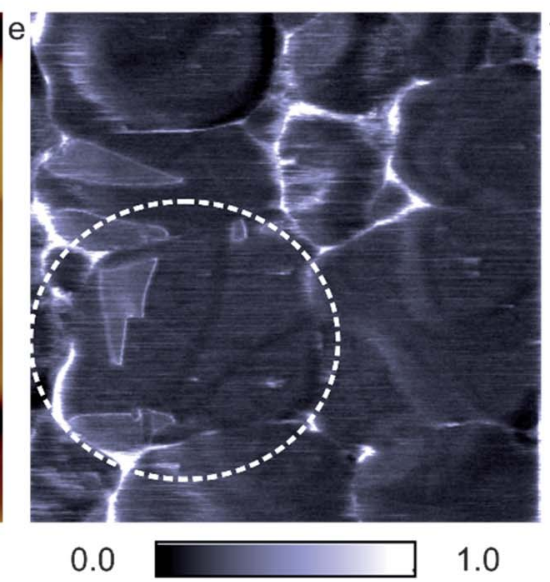

VPFM amplitude (a.u.)

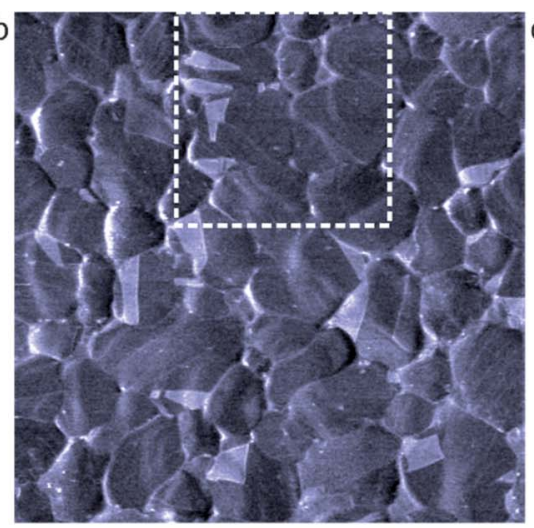

0.0

1.0

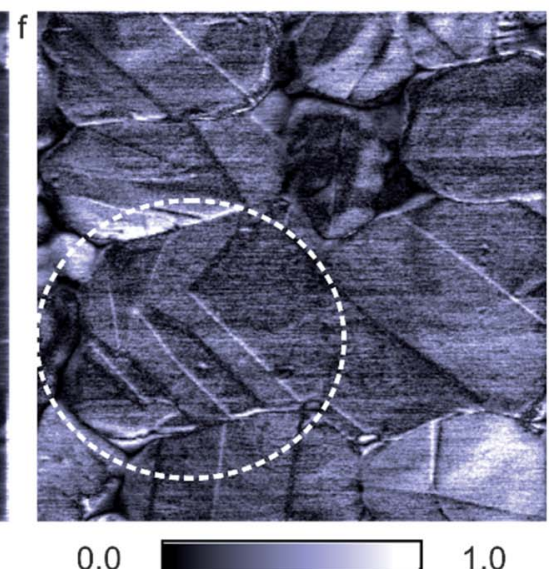

Fig. $5 \mathrm{MAPb}_{3}$ grains exhibit both out-of-plane and in-plane polarized domains after 2 min of thermal annealing. (a) Topography, (b) VPFM and (c) LPFM images. (d-f) The magnifications show the displacement of out-of-plane polarized domains by in-plane polarized domains. On the marked grain, an indentation of an out-of-plane polarized domain by the tip of an in-plane polarized domain that grew from the grain boundary becomes visible. Hence, this individual grain exhibits three different polarization orientations: besides the out-of-plane polarized domains, two alternating in-plane polarization orientations are clearly distinguishable in VPFM and LPFM. 
phase image (Fig. 6b) before the second annealing reveals inplane polarization on most grains, but with non-uniform domain patterns. At position I, the domains are needleshaped as described in Section 2.3. At least one of these needle-shaped domains extends over the adjacent grain boundary, facing other lateral domains perpendicularly. At positions II and III, the domain patterns are confined to individual grains. While the domains at position II vary in width,
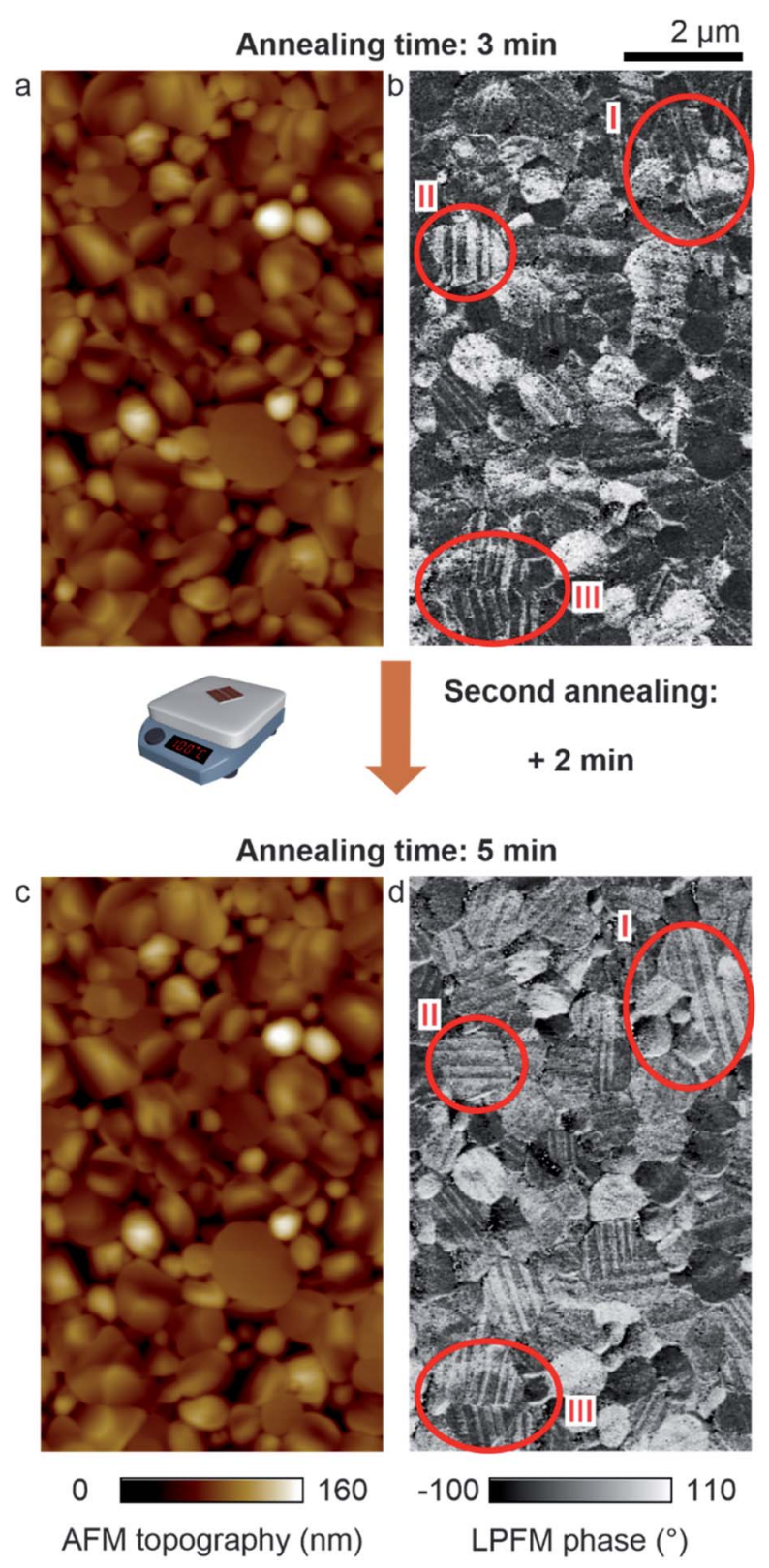

Fig. 6 Formation of lateral domains with alternating in-plane polarization upon thermal annealing. (a) Topography of a representative $\mathrm{MAPb}_{3}$ sample annealed for $3 \mathrm{~min}$. (b) The LPFM phase shows striped domains. (c) Upon additional annealing for $2 \mathrm{~min}$, the topography does not change, but some domains are strongly modified: (d) the LPFM phase image depicts domains that expand over grain boundaries (I), a rotation of domain patterns by $90^{\circ}$ (II) or domains that retain their shapes, dimensions and positions (III). the lamellar domains at position III are rather evenly distributed.

After the second annealing step of $2 \mathrm{~min}$, some changes to the pattern of lateral domains become visible in the LPFM phase image in Fig. 6d. The initial needle-like domains at position I (Fig. 6b) grow and expand to uniformly alternating domains, notably extending over several grains (Fig. 6d). Moreover, we observed the formation of new lateral domain patterns, such as the replacement of domains and domain walls by perpendicular domains (positions I + II).

Both the formation of new domains and the modification of domains likely appear because of energetic optimization for both mechanical stress and electrostatic forces, similar to other ferroelectrics. ${ }^{9}$ Mediated by thermal annealing, this evolution of laterally polarized domains continues until a thermodynamically stable configuration of alternating domains is reached (sample 5, Fig. 1). At position III, the grain already exhibited a uniform domain pattern before the second annealing step. This domain pattern persisted after the second annealing. We note that the second annealing step for $2 \mathrm{~min}$ did not affect the topography (Fig. 6c).

From these observations, we conclude that the domains with vertical polarization may well influence the initial formation of lateral domains and their shapes. At a later stage, when these vertically polarized domains are extinct, we expect grain boundaries and crystal defects to control the formation of ferroelectric domains, as it is also known from other, inorganic perovskites. $^{\mathbf{5 0 , 5 1}}$ This interpretation is supported by a distinct angle of $45^{\circ}$ between lateral domain walls and the grain boundaries (Fig. 5e and $\mathrm{S} 4 \mathrm{~b} \dagger$ ). ${ }^{22}$ Under the assumption that grain boundaries preferentially form along the (110) and $\left(11^{*} 0\right)$ crystal planes in $\mathrm{MAPbI}_{3}$ (i.e. the basic planes of the MA-cation), this angle would signify lateral domain walls forming along the [100] and [010] directions. ${ }^{22}$

The reappearance of domain walls at position III upon heating above $T_{\mathrm{C}}$ and subsequently cooling down below $T_{\mathrm{C}}$ indicates an already energetically stable configuration, but also pinning at crystal defects and grain boundaries as well as clamping due to lateral stress. The latter may either originate from defect "healing" ${ }^{52}$ within the grains due to annealing or could stem from uniaxial forces exerted by neighboring grains. ${ }^{53,54}$ In polycrystalline thin-films, mechanical stress is a generic feature that occurs during grain growth and the formation of thin-films. ${ }^{34}$ Consequently, stress induced pinning may suppress domain modifications and hence force lattice distortions. ${ }^{19,54}$

Our study reveals that the annealing procedure acts as a driving force for the transformation of ferroelectric domains. During annealing the aspect ratio of grains changes significantly: grains grow to lateral diameters of several microns while their thickness remains constant at $300 \mathrm{~nm}$. Initially, several polarization orientations coexist but mainly in-plane polarization remains after extended annealing. The domains evolve in $\mathrm{MAPbI}_{3}$ thin-films until stable domain patterns of alternating in-plane polarization exist. Once the polarization was switched to in-plane orientation, in our study, annealing has never triggered reappearance of any vertical domains. 
a
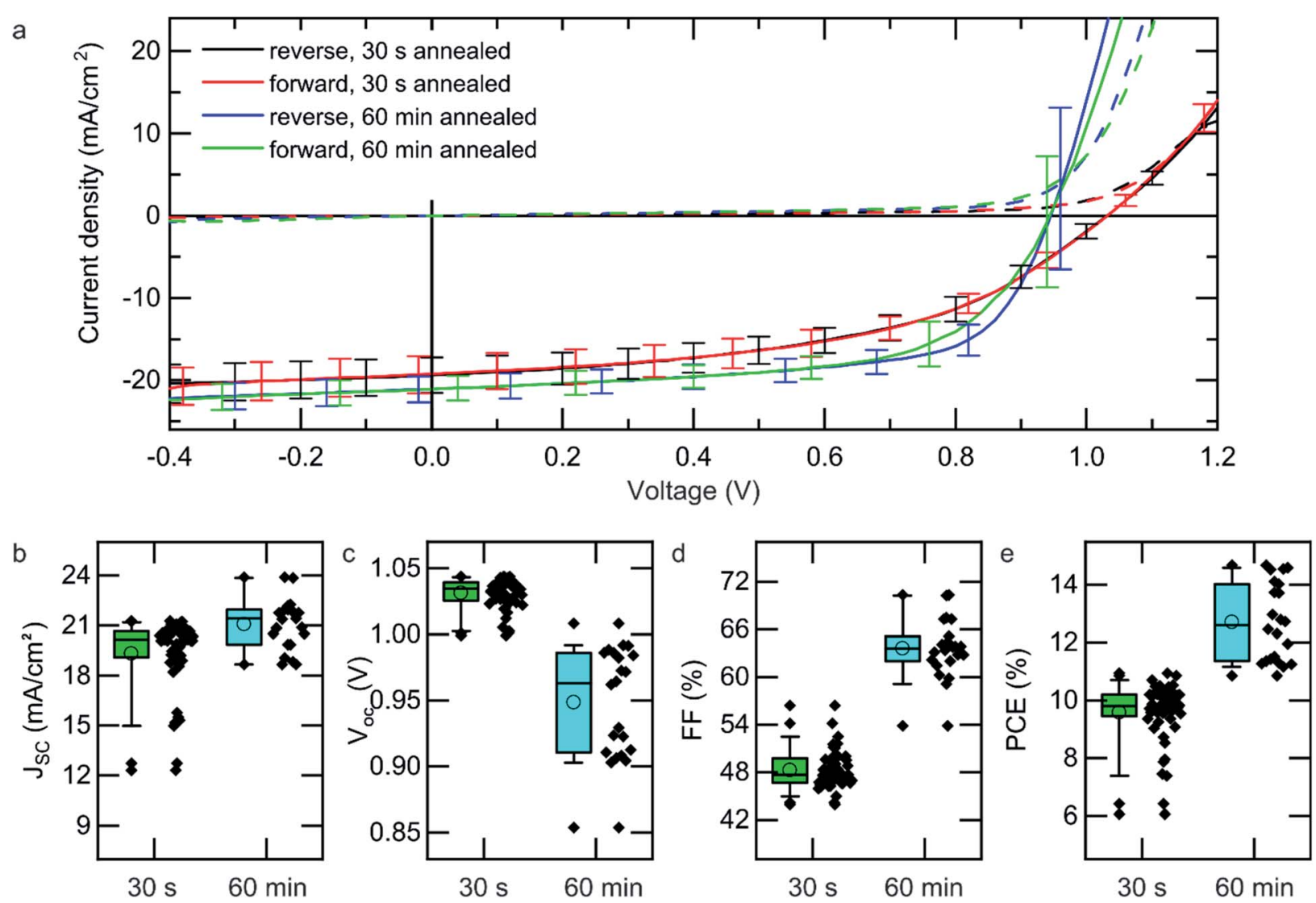

Fig. 7 Solar cell performance in dependence of the perovskite layer annealing time. (a) Averaged J-V curves of solar cells comprising MAPbl 3 thin-films that were annealed for $30 \mathrm{~s}$ during fabrication. These solar cells show reduced fill factors but higher $V_{O C}$ in comparison to solar cells comprising $\mathrm{MAPbl}_{3}$ thin-films that were annealed for $60 \mathrm{~min}$. (Dashed lines represent dark curves of the solar cells.) (b-e) Characteristic solar cell parameters extracted from reverse and forward $J-V$ scans.

\subsection{Correlation of ferroelectric domain polarization and solar cell properties}

To date, the extent to which ferroelectric domains influence the solar cell performance remains yet to be investigated, since a fundamental understanding of ferroelectric domain formation had been missing. With the toolbox above that allows the targeted formation of domains with in-plane and out-of-plane polarization, we now have the experimental means to correlate the domain polarization and the performance of $\mathrm{MAPbI}_{3}$ solar cells. For this study, we fabricated solar cells comprising polycrystalline $\mathrm{MAPbI}_{3}$ layers that were deposited as described above. One set of solar cells was annealed for $30 \mathrm{~s}$, after which the MAI/PbI ${ }_{2}$-to-MAPbI ${ }_{3}$ conversion process was completed, and another set of samples was annealed for $60 \mathrm{~min}$ right after $\mathrm{MAPbI}_{3}$ deposition, both producing reproducible domain patterns. The former set exhibits notable out-of-plane domain polarization, the latter predominant in-plane domain polarization. To complete the solar cells, transport layers of $[6,6]-$ phenyl $\mathrm{C}_{71}$-butyric acid methyl ester $\left(\mathrm{PC}_{71} \mathrm{BM}\right)$ and bathocuproine (BCP) were deposited from solution and a silver top electrode was sublimed in high vacuum. After the deposition of the perovskite layer, the solar cells were not annealed anymore to ensure stable domain configurations. During vacuum sublimation of the silver electrode, the device temperature did not exceed $40{ }^{\circ} \mathrm{C}$ and hence remained well below $T_{\mathrm{C}}$.

2.2.1 Solar cell performance. The solar cells yield peakPCEs of $15 \%$ which is comparable to previous literature reports on devices with similar architectures, featuring PEDOT:PSS as a hole-transport layer atop the bottom ITO electrode. ${ }^{55,56}$ Fig. 7 a depicts the averaged current density-voltage $(J-V)$ characteristics of both sets of solar cells, measured in forward and reverse direction, and Fig. 7b-e shows a statistical analysis of the data.

The black and red curves represent the backward and forward sweeps of 26 solar cells comprising $\mathrm{MAPbI}_{3}$ layers that were annealed for $30 \mathrm{~s}$ and exhibit large domains with out-ofplane polarization as exemplified in ESI Fig. S8. $\dagger$ The blue and green curves correspond to 11 solar cells with $\mathrm{MAPbI}_{3}$ layers that were thermally annealed for $60 \mathrm{~min}$ and possess striped lateral domains. Strikingly, the two sets of solar cells exhibit significantly different fill factors (FFs) and hence device performance: while solar cells with out-of-plane polarization components exhibit lower performance (average FF $<50 \%$, PCE $=10 \%)$, the solar cells with alternating in-plane polarized domains possess average $\mathrm{FF}=65 \%$ (Fig. $7 \mathrm{~d}$ ) and power conversion efficiencies (PCEs) of 13\% (see Fig. 7e). 
a

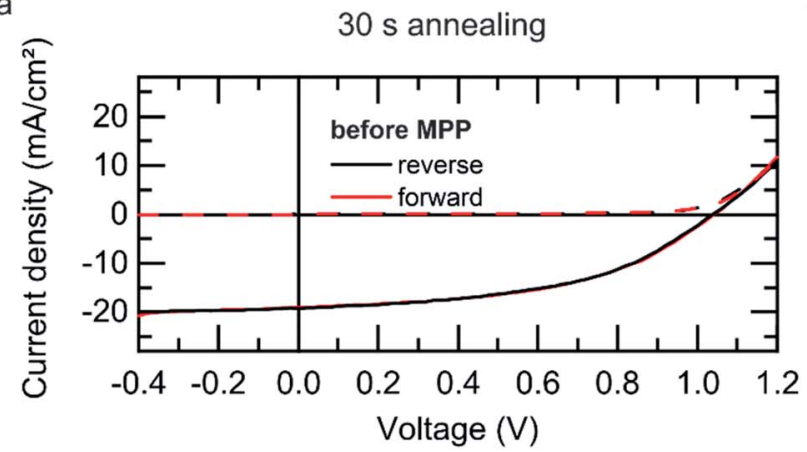

b

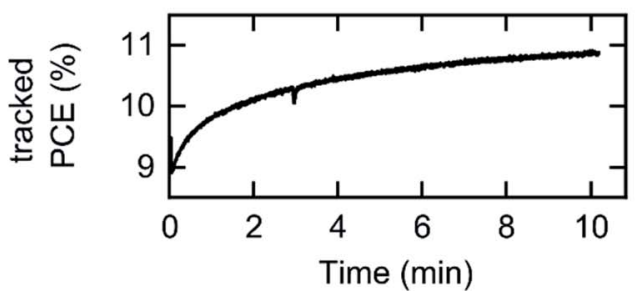

$\mathrm{C}$

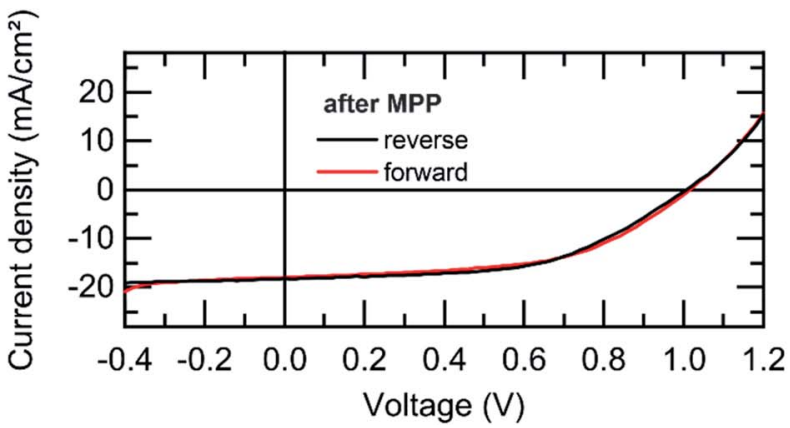

d

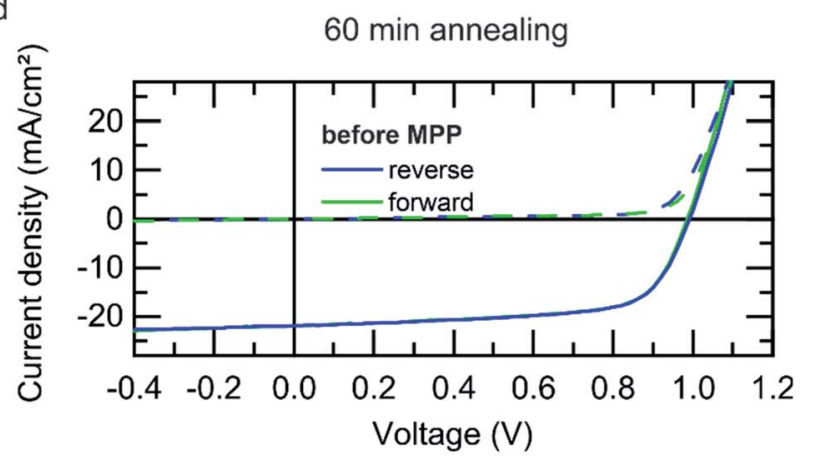

e
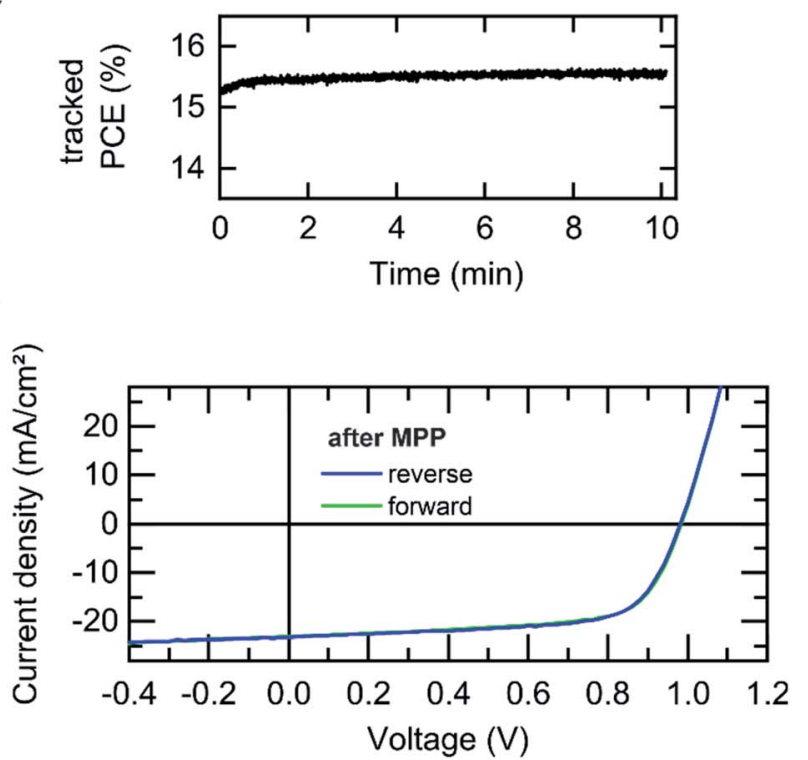

Fig. 8 MPP tracking under 1 sun illumination. (a) Initial J-V curves of a solar cell that was annealed for $30 \mathrm{~s}$. (b) The PCE increases significantly during MPP measurement. (c) After MPP-tracking, the $J-V$ curves of the solar cell revert back to their initial shape. (d-f) After 60 min of thermal annealing, the PCE of a solar cell remains constant during MPP-tracking and the $J-V$ curves persisted.

Notably, the influence of the domain polarization on the solar cell performance may be overlaid by other microstructural contributions such as grain growth during ongoing annealing and a concomitant change of grain boundary densities and defect states. ${ }^{57-59}$ Although these effects are partly difficult to disentangle experimentally, our observations very much resemble earlier theoretical studies on the influence of ferroelectric polarization on the solar cell performance. ${ }^{12}$ It was predicted that domains with alternating in-plane polarizations and their concomitant electrical fields assist with the spatial separation of charges and produce pathways for electrons and holes to the respective electrodes. ${ }^{11,12}$ Consequently, ShockleyRead-Hall recombination could be reduced, which in turn would improve the PCE of the solar cell due to an enhanced fill factor. ${ }^{12}$ Likewise, these simulations predicted that any out-ofplane polarization would not contribute to an improvement in the solar cell performance, producing only moderate efficiencies. Other earlier experimental works have reported that the performance of optimized solar cells decreases upon heating to above $T_{\mathrm{C}}{ }^{60}$ where ferroelectric domains would usually disappear. The roles of ionic charges in the formation of domains has been discussed in our earlier work. ${ }^{22}$

In addition, we observe an average open-circuit voltage $V_{\mathrm{OC}}=$ $1031 \pm 11 \mathrm{mV}$ in the set of solar cells with out-of-plane polarization, which is $80 \mathrm{mV}$ higher than the $V_{\mathrm{OC}}$ of solar cells comprising domains with in-plane polarization $\left(V_{\mathrm{OC}}=948 \pm 42\right.$ $\mathrm{mV})$. Interestingly, this difference in $V_{\mathrm{OC}}$ is close to the $50 \mathrm{mV}$ difference of the CPD between surfaces of domains with out-ofplane polarization and surfaces of domains with no out-ofplane polarization, which we discussed in Section 2.2. In light of the KPFM measurements in Section 2.2, we conclude that the $V_{\mathrm{OC}}$ is modified not only by diverse crystal facets that modify the interface between $\mathrm{MAPbI}_{3}$ and the charge carrier transport layers, ${ }^{61,62}$ but also by the screening surface charges of the vertical ferroelectric domains.

2.2.2 Solar cell performance vs. time. Finally, the principal studies on the thermal instability of vertically polarized domains in $\mathrm{MAPbI}_{3}$ thin-films in Section 2.3 has led us to examine the stability of solar cells comprising domains with out-of-plane polarization. Fig. 8a depicts the $J-V$ curves in reverse and forward scanning direction (black and red) of 
a representative solar cell with a $\mathrm{MAPbI}_{3}$ layer that was annealed for $30 \mathrm{~s}$ and hence contains out-of-plane polarized domains. In accordance with our previous experiment, the low initial fill factor of the solar cell produces a moderate PCE of 9\%. In order to examine the device stability, we exposed the solar cell to 1 sun (irradiance $E=1000 \mathrm{~W} \mathrm{~m}^{-2}, \mathrm{AM} 1.5$ ) and tracked the maximum power point (MPP). Each voltage level was held for $300 \mathrm{~ms}$ and the respective PCE was calculated from the measured power output. Fig. 8b shows an immediate increase of the PCE at the beginning of the MPP-tracking. During 10 minutes of continuous measurements, the PCE steadily increases and reaches almost $11 \%$, which corresponds to an improvement of more than $20 \%$ relative to the initial PCE. In contrast, solar cells with alternating lateral domains, which were exposed to a thermal annealing for $60 \mathrm{~min}$, possess a rather constant (and higher) PCE during MPP-tracking and the initial $J-V$ curves very much persisted afterwards (Fig. 8d-f).

Remarkably, $35 \mathrm{~s}$ after discontinuation of the 10 min of MPP tracking, the $J-V$ curves of the solar cell in Fig. 8c reverted to about their initial shape. Only after extending the MPP tracking to $60 \mathrm{~min}$, the changed $J-V$ curves and hence the improved PCE persisted (ESI Fig. S9 $\dagger$ ) showing reduced $V_{\text {OC }}$, and an enhanced fill factor. Notably, both types of solar cells in Fig. 8 show low hysteresis before and after MPP tracking which may indicate that ionic effects play a secondary role here. According to our experiments described above, the improvement of the PCE during MPP-tracking may well have its origin in a change of polarization orientation. Within the first $10 \mathrm{~min}$ of the experiment, the changes of the polarization direction may be rather small, and hence it reverts to its original orientation after discontinuation of the MPP tracking, e.g. mediated by pinning at defects or layer interfaces. A gradual increase of the polarization modulation for a longer period of MPP tracking may eventually bring the sample into a new equilibrium state from where the reverse process appears energetically unfavorable. The activation energy to overcome this tipping point may well stem from sample heating by illumination or from the electrical energy supply. Although this interpretation appears logical in light of the previous experiments, notably, tracking the change in polarization orientation is impossible with the experimental methods used here, as the top metal electrode conceals the sample region of interest. Removing the electrode or cleaving the sample would very likely result in mechanical strain that, by itself, can change the polarization orientation.

\section{Conclusions}

Our experimental study demonstrates how ferroelectric domains evolve upon thermal annealing, which is a common fabrication step of $\mathrm{MAPbI}_{3}$ solar cells. Within seconds to minutes, thermal annealing of $\mathrm{MAPbI}_{3}$ thin-films triggers the transformation of coexisting out-of-plane and in-plane polarizations towards a predominant in-plane polarization. We tracked this transformation by studying distinct contrasts in VPFM and LPFM imaging. During this transformation, we occasionally observed at least three different polarization orientations within individual grains, supporting the case for ferroelectricity of $\mathrm{MAPbI}_{3}$. Vertical polarization components modulate the surface potential (CPD) in order to compensate internal electrical fields as visible in FM-KPFM.

In recent years, many reports focused on the influence of the manufacturing processes of $\mathrm{OMH}$ perovskite thin-films on the performance of solar cells. In particular, the layers underneath, ${ }^{63,64}$ additives to the precursor solutions, ${ }^{65-67}$ solvent vapor annealing, ${ }^{55,68,69}$ variations of the annealing temperature ${ }^{38,70}$ and the annealing duration ${ }^{71}$ have been reported to affect the thinfilm formation and to determine the power conversion efficiency. The correlation of ferroelectric domain polarization and device performance in this work suggests that the configuration and evolution of ferroelectric domains should be considered as an additional critical mechanism affecting solar cell characteristics. Solar cells with alternating lateral domains comprise best fill factors and efficiencies, whereas solar cells with shortlyannealed $\mathrm{MAPbI}_{3}$ thin-films and vertical domains exhibit only moderate performance. Not least, solar cells with out-of-plane polarization presented themselves less stable during electrical characterization. This observation is critically important for any type of $J-V$ measurements or in particular MPP-tracking. Notably, intrinsic conductivity, photoconductivity and ionic conductivity also change during thermal annealing and hence may also affect the solar cell properties. While it is difficult, if not impossible, to disentangle these effects, the agreement of this experimental study with earlier theoretic discussions and drift-diffusion modeling within ferroelectric domain assemblies is intriguing. ${ }^{\mathbf{1 2}}$

\section{Experimental}

\subsection{Sample fabrication}

The polycrystalline $\mathrm{MAPbI}_{3}$ thin-films were fabricated following our earlier works and characterized under inert atmosphere. ${ }^{\mathbf{1 4 , 1 9}}$ The indium tin oxide (ITO) coated substrates were cleaned with acetone and isopropanol in an ultrasonic bath, after which they were exposed to an oxygen plasma for $2 \mathrm{~min}$ and transferred into a glovebox. Poly(3,4-ethylenedioxythiophene): poly(4-styrenesulphonate) (PEDOT:PSS, VPAI 4083, Heraeus Clevios, diluted with ethanol $1: 3 \mathrm{v} / \mathrm{v}$ ) was blade-coated (Zehntner ZUA-2000) atop the ITO, and the substrates were thermally annealed for $10 \mathrm{~min}$ at $140{ }^{\circ} \mathrm{C}$. Lead iodide $\left(\mathrm{PbI}_{2}\right.$, Sigma Aldrich or Alfa Aesar (sample 5, Fig. 1); both $\mathrm{PbI}_{2}$ yield the same grain sizes and domain patterns, ESI Fig. S10†) was dissolved in dimethyl sulfoxide (DMSO, $250 \mathrm{mg} \mathrm{mL}^{-1}$ ) and 1chloronaphthalene was added to the solution $(100: 1 \mathrm{v} / \mathrm{v})$. Then, the $\mathrm{PbI}_{2}$ solution was blade-coated onto the PEDOT:PSS layer $\left(4 \mathrm{~mm} \mathrm{~s}^{-1}\right.$, at $60{ }^{\circ} \mathrm{C}$, gap $\left.70 \mu \mathrm{m}\right)$ and quenched with a nitrogen stream. In a second step, a solution of methylammonium iodide (MAI, Lumtec Ltd.) and methylammonium chloride (MACl, MAI : MACl 9 : $1 \mathrm{wt} / \mathrm{wt}$, in isopropanol $40 \mathrm{~g} \mathrm{~L}^{-1}$ ) was spin cast (1350 rpm) to convert the precursors into a $300 \mathrm{~nm}$ $\mathrm{MAPbI}_{3}$ thin-film. All samples were initially annealed $\left(100{ }^{\circ} \mathrm{C}, 5\right.$ s) to warrant a complete and rapid conversion into perovskite structure. Any further annealing as described above was accomplished under Petri dishes (volume approx. $5 \mathrm{~cm}^{3}$ ) for the specified annealing durations. To fabricate solar cells, the 
samples were completed by spin casting layers of [6,6]-phenyl $\mathrm{C}_{71}$-butyric acid methyl ester $\left(\mathrm{PC}_{71} \mathrm{BM}\right.$ in chlorobenzene) and bathocuproine (BCP in ethanol) on top of $\mathrm{MAPbI}_{3}$. A silver top electrode was sublimed in high vacuum.

\subsection{Annealing conditions}

Fig. 1: sample 1: $5 \mathrm{~s}$ no Petri dish; samples 2, 3, 4 and 5: $30 \mathrm{~s}$, 60 s, 3 min, 60 min with Petri dish. Fig. 2: (a) 5 s no Petri dish; (b) $30 \mathrm{~s}$ with Petri dish, (c) $60 \mathrm{~min}$ with Petri dish. Fig. 3: $5 \mathrm{~s}$ and then $60 \mathrm{~s}$ with Petri dish. Fig. 4: $(\mathrm{a}+\mathrm{b}) 5 \mathrm{~s}$ and then $60 \mathrm{~s}$ with Petri dish; $(\mathrm{c}+\mathrm{d})$ additional annealing for $60 \mathrm{~s}$ with Petri dish (total annealing: $120 \mathrm{~s}$ ). Fig. 5: $5 \mathrm{~s}$ and then 2 min with Petri dish. Fig. 6: $(\mathrm{a}+\mathrm{b}) 5 \mathrm{~s}$ and then 3 min with Petri dish; $(\mathrm{c}+\mathrm{d})$ additional annealing for 2 min with Petri dish (total annealing: 5 min). Fig. 7 and 8: annealing like samples 2 and 5 in Fig. 1.

\subsection{Atomic force microscopy}

The atomic force microscopy (AFM) measurements were performed on a Dimension Icon (Bruker) under nitrogen atmosphere with platinum-iridium coated conductive tips (SCM-PIC, spring constant $k=0.1 \mathrm{~N} \mathrm{~m}^{-1}$ ). In order to enhance the resolution and reduce the signal-to-noise ratio, contact resonance amplification was used for VPFM and LPFM, and the driving AC frequencies were set a few $\mathrm{kHz}$ below the respective contact resonance. Forces in the range of 3-10 $\mathrm{nN}$ and moderate ACbias amplitudes of $1000-1500 \mathrm{mV}$ were applied in order to minimize damages to the thin-film samples and enable repetitive measurements of several PFM micrographs at the same sample position. The PFM amplitude and phase scales were chosen for optimal contrast. The contact resonance enhancement impedes a reliable absolute calibration of the PFM data, and the scale bars were therefore set to arbitrary units for both LPFM and VPFM amplitudes. For FM-KPFM, platinum-iridium coated tips (SCM-PIT-V2, spring constant $k=3 \mathrm{~N} \mathrm{~m}^{-1}$ ) and a drive amplitude of $3000 \mathrm{mV}$ were used. All PFM and KPFM scans were conducted in the dark without additional DC biases.

\subsection{Optoelectronic characterization}

The current density-voltage $(J-V)$ curves of the solar cells were measured in inert atmosphere with a Keithley source measurement unit in the dark and under illumination from a solar simulator (Sciencetech Lightline, AM 1.5, $1000 \mathrm{~W} \mathrm{~m}^{-2}$, calibrated with a Si reference solar cell). The scan speed in reverse $(1.2 \mathrm{~V} \rightarrow-0.4 \mathrm{~V})$ and forward $(-0.4 \mathrm{~V} \rightarrow 1.2 \mathrm{~V})$ direction was $100 \mathrm{mV} \mathrm{s}^{-1}$. During MPP tracking, the voltage was held for $300 \mathrm{~ms}$ and the PCE was calculated from the respective current. The $J-V$ curves were measured again $30-60 \mathrm{~s}$ after completion of the MPP tracking.

\subsection{X-ray diffraction}

Powder diffraction data were collected on a D8 Advance (Bruker AXS) equipped with an energy discriminating LYNXEYE XE-T $1 \mathrm{D}$ detector using $\mathrm{Cu} \mathrm{K} \alpha$ radiation in a $\theta-2 \theta$ scan in the range from $5^{\circ}$ to $60^{\circ} 2 \theta$ with a step size of $0.010^{\circ}$ and measurement time of $4 \mathrm{~s}$ per step. Due to their sensitivity to oxygen and moisture, the samples were packed into a sealed sample holder inside a glove box under nitrogen atmosphere, and instantly measured after unloading. The texture axis was assumed to be perpendicular to the surface of the sample, permitting the use of sample rotation $\left(30^{\circ} \mathrm{s}^{-1}\right)$ to reduce the effects of particle counting statistics.

\section{Author contributions}

T. L., H. R. and A. C. have conceived the experiments and jointly written the manuscript. T. L. and H. R. have carried out the experiments. F. A. has contributed the XRD measurements and their interpretation. M. J. H. has advised on data interpretation and ferroelectric properties. A. C. has supervised the work. All authors have discussed the data and approved the manuscript.

\section{Conflicts of interest}

There are no conflicts to declare.

\section{Acknowledgements}

The authors acknowledge funding by the Carl Zeiss Foundation (project KeraSolar) and support by the Helmholtz Programme "Science and Technology of Nanosystems" (STN). T. L. acknowledges funding by the Landesgraduiertenförderung Baden-Württemberg. We thank Simon Petrick for performing the grain size analysis and Alexander D. Schulz for fruitful discussions.

\section{References}

1 M. A. Green, E. D. Dunlop, J. Hohl-Ebinger, M. Yoshita, N. Kopidakis and A. W. Y. Ho-Baillie, Prog. Photovoltaics, 2020, 28, 3-15.

2 C. Wehrenfennig, G. E. Eperon, M. B. Johnston, H. J. Snaith and L. M. Herz, Adv. Mater., 2014, 26, 1584-1589.

3 L. M. Herz, ACS Energy Lett., 2017, 2, 1539-1548.

4 J. M. Frost, K. T. Butler and A. Walsh, APL Mater., 2014, 2, 081506.

5 A. M. A. Leguy, J. M. Frost, A. P. McMahon, V. G. Sakai, W. Kockelmann, C. Law, X. Li, F. Foglia, A. Walsh, B. C. O'Regan, J. Nelson, J. T. Cabral and P. R. F. Barnes, Nat. Commun., 2015, 6, 7124.

6 A. Stroppa, C. Quarti, F. De Angelis and S. Picozzi, J. Phys. Chem. Lett., 2015, 6(12), 2223-2231.

7 M. E. Lines and A. M. Glass, Principles and Applications of Ferroelectrics and Related Materials, Oxford University Press, Oxford, 1977.

8 B. Jaffe, W. R. Cook Jr and H. Jaffe, Piezoelectric Ceramics, Academic Press, London, 1971.

9 D. Damjanovic, Rep. Prog. Phys., 1998, 61, 1267.

10 J. M. Frost, K. T. Butler, F. Brivio, C. H. Hendon, M. van Schilfgaarde and A. Walsh, Nano Lett., 2014, 14(5), 25842590.

11 T. S. Sherkar and L. J. A. Koster, Phys. Chem. Chem. Phys., 2016, 18, 331. 
12 D. Rossi, A. Pecchia, M. Auf der Maur, T. Leonhard, H. Röhm, M. J. Hoffmann, A. Colsmann and A. Di Carlo, Nano Energy, 2018, 48, 20-26.

13 I. M. Hermes, S. A. Bretschneider, V. W. Bergmann, D. Li, A. Klasen, J. Mars, W. Tremel, F. Laquai, H.-J. Butt, M. Mezger, R. Berger, B. J. Rodriguez and S. A. L. Weber, J. Phys. Chem. C, 2016, 120, 5724-5731.

14 H. Röhm, T. Leonhard, M. J. Hoffmann and A. Colsmann, Energy Environ. Sci., 2017, 10, 950.

15 C. Harnagea, A. Pignolet, M. Alexe and D. Hesse, Integr. Ferroelectr., 2004, 60, 101-110.

16 L. Collins, Y. Liu, O. S. Ovchinnikova and R. Proksch, ACS Nano, 2019, 13(7), 8055-8066.

17 R. K. Vasudevan, N. Balke, P. Maksymovych, S. Jesse and S. V. Kalinin, Appl. Phys. Rev., 2017, 4, 021302.

18 C. Eames, J. M. Frost, P. R. F. Barnes, B. C. O'Regan, A. Walsh and M. S. Islam, Nat. Commun., 2015, 6, 7497.

19 T. Leonhard, A. D. Schulz, H. Röhm, S. Wagner, F. J. Altermann, W. Rheinheimer, M. J. Hoffmann and A. Colsmann, Energy Technol., 2019, 7, 1800989.

20 H. Röhm, T. Leonhard, A. D. Schulz, S. Wagner, M. J. Hoffmann and A. Colsmann, Adv. Mater., 2019, 31, 1806661.

21 J. Breternitz, F. Lehmann, S. A. Barnett, H. Nowell and S. Schorr, Angew. Chem., 2020, 132, 432-436.

22 H. Röhm, T. Leonhard, M. J. Hoffmann and A. Colsmann, Adv. Funct. Mater., 2020, 30, 1908657.

23 Y. Rakita, O. Bar-Elli, E. Meirzadeh, H. Kaslasi, Y. Peleg, G. Hodes, I. Lubomirsky, D. Oron, D. Ehre and D. Cahen, Proc. Natl. Acad. Sci. U. S. A., 2017, 114(28), E5504-E5512.

24 Z. Fan, J. Xiao, K. Sun, L. Chen, Y. Hu, J. Ouyang, K. P. Ong, K. Zeng and J. Wang, J. Phys. Chem. Lett., 2015, 6, 1155-1161.

25 W. T. H. Koch, R. Munser, W. Ruppel and P. Würfel, Solid State Commun., 1975, 17(7), 847-850.

26 R. von Baltz and W. Kraut, Phys. Rev. B: Condens. Matter Mater. Phys., 1981, 23(10), 5590-5596.

27 E. Strelcov, Q. Dong, T. Li, J. Chae, Y. Shao, Y. Deng, A. Gruverman, J. Huang and A. Centrone, Sci. Adv., 2017, 3, e1602165.

28 G. A. MacDonald, C. M. Heveran, M. Yang, D. Moore, K. Zhu, V. L. Ferguson, J. P. Killgore and F. W. DelRio, ACS Appl. Mater. Interfaces, 2017, 9, 33565-33570.

29 Y. Liu, L. Collins, R. Proksch, S. Kim, B. R. Watson, B. Doughty, T. R. Calhoun, M. Ahmadi, A. V. Ievlev, S. Jesse, S. T. Retterer, A. Belianinov, K. Xiao, J. Huang, B. G. Sumpter, S. V. Kalinin, B. $\mathrm{Hu}$ and O. S. Ovchinnikova, Nat. Mater., 2018, 17, 1013-1019.

30 I. M. Hermes, A. Best, L. Winkelmann, J. Mars, S. M. Vorpahl, M. Mezger, L. Collins, H.-J. Butt, D. S. Ginger, K. Koynov and S. A. L. Weber, Energy Environ. Sci., 2020, 13, 4168-4177.

31 S. M. Vorpahl, R. Giridharagopal, G. E. Eperon, I. M. Hermes, S. A. L. Weber and D. S. Ginger, ACS Appl. Energy Mater., 2018, 1, 1534-1539.

32 B. Huang, G. Kong, E. N. Esfahani, S. Chen, Q. Li, J. Yu, N. Xu, Y. Zhang, S. Xie, H. Wen, P. Gao, J. Zhao and J. Li, npj Quantum Mater., 2018, 3, 30.
33 A. D. Schulz, H. Röhm, T. Leonhard, S. Wagner, M. J. Hoffmann and A. Colsmann, Nat. Mater., 2019, 18, 1050.

34 G. Arlt, Ferroelectrics, 1990, 104(1), 217-227.

35 A. Poglitsch and D. Weber, J. Chem. Phys., 1987, 87, 11.

36 Z. Xiao, C. Bi, Y. Shao, Q. Dong, Q. Wang, Y. Yuan, C. Wang,

Y. Gao and J. Huang, Energy Environ. Sci., 2014, 7, 2619.

37 C. Bi, Y. Shao, Y. Yuan, Z. Xiao, C. Wang, Y. Gao and J. Huang, J. Mater. Chem. A, 2014, 2, 18508.

38 W. Nie, H. Tsai, R. Asadpour, J.-C. Blancon, A. J. Neukirch, G. Gupta, J. J. Crochet, M. Chhowalla, S. Tretiak, M. A. Alam, H.-L. Wang and A. D. Mohite, Science, 2015, 347(6221), 522-525.

39 M. Ralaiarisoa, Y. Busby, J. Frisch, I. Salzmann, J.-J. Pireaux and N. Koch, Phys. Chem. Chem. Phys., 2017, 19, 828.

40 N. J. Jeon, J. H. Noh, Y. C. Kim, W. S. Yang, S. Ryu and S. I. Seok, Nat. Mater., 2014, 13, 897-903.

41 M. Saliba, J.-P. Correa-Baena, C. M. Wolff, M. Stolterfoht, N. Phung, S. Albrecht, D. Neher and A. Abate, Chem. Mater., 2018, 30, 4193-4201.

42 A. Gruverman, M. Alexe and D. Meier, Nat. Commun., 2019, 10, 1661.

43 E. Soergel, J. Phys. D: Appl. Phys., 2011, 44, 464003.

44 S. V Kalinin, Y. Kim, D. D Fong and A. N. Morozovska, Rep. Prog. Phys., 2018, 81, 036502.

45 S. Sadewasser, T. Glatzel, M. Rusu, A. Jäger-Waldau and M. C. Lux-Steiner, Appl. Phys. Lett., 2002, 80, 2979.

46 R. C. Bradt and G. S. Ansell, J. Am. Ceram. Soc., 1969, 52, 192198.

47 W. A. Schulze and K. Ogino, Ferroelectrics, 1988, 87, 361-377. 48 I. S. Zheludev, Domain Structure of Ferroelectrics and Antiferroelectrics, in Physics of Crystalline Dielectrics, Springer, Boston, 1971.

49 A. Hershkovitz, F. Johann, M. Barzilay, A. H. Avidor and Y. Ivry, Acta Mater., 2020, 187, 186-190.

50 P. V. Lambeck and G. H. Jonker, Ferroelectrics, 1978, 22, 729731.

51 B. J. Rodriguez, Y. H. Chu, R. Ramesh and S. V. Kalinin, Appl. Phys. Lett., 2008, 93, 142901.

52 F. Wang, S. Bai, W. Tress, A. Hagfeldt and F. Gao, npj Flexible Electron., 2018, 2, 22.

53 G. Arlt, J. Mater. Sci., 1990, 25, 2655-2666.

54 S. Jariwala, H. Sun, G. W. P. Adhyaksa, A. Lof, L. A. Muscarella, B. Ehrler, E. C. Garnett and D. S. Ginger, Joule, 2019, 3(12), 3048-3060.

55 Z. Xiao, Q. Dong, C. Bi, Y. Shao, Y. Yuan and J. Huang, Adv.Mater., 2014, 26, 6503-6509.

56 P. Fassl, V. Lami, A. Bausch, Z. Wang, M. T. Klug, H. J. Snaith and Y. Vaynzof, Energy Environ. Sci., 2018, 11, 3380.

57 G. W. P. Adhyaksa, S. Brittman, H. Abolins, A. Lof, X. Li, J. D. Keelor, Y. Luo, T. Duevski, R. M. A. Heeren, S. R. Ellis, D. P. Fenning and E. C. Garnett, Adv. Mater., 2018, 30, 1804792.

58 R. Ciesielski, F. Schäfer, N. F. Hartmann, N. Giesbrecht, T. Bein, P. Docampo and A. Hartschuh, ACS Appl. Mater. Interfaces, 2018, 10, 7974-7981. 
59 N. Phung, A. Al-Ashouri, S. Meloni, A. Mattoni, S. Albrecht, E. L. Unger, A. Merdasa and A. Abate, Adv. Energy Mater., 2020, 10, 1903735.

60 J. A. Schwenzer, L. Rakocevic, R. Gehlhaar, T. Abzieher, S. Gharibzadeh, S. Moghadamzadeh, A. Quintilla, B. S. Richards, U. Lemmer and U. W. Paetzold, ACS Appl. Mater. Interfaces, 2018, 10(19), 16390-16399.

61 S. A. L. Weber, I. M. Hermes, S.-H. Turren-Cruz, C. Gort, V. W. Bergmann, L. Gilson, A. Hagfeldt, M. Graetzel, W. Tress and R. Berger, Energy Environ. Sci., 2018, 11, 2404. 62 S. Y. Leblebici, L. Leppert, Y. Li, S. E. Reyes-Lillo, S. Wickenburg, E. Wong, J. Lee, M. Melli, D. Ziegler, D. K. Angell, D. F. Ogletree, P. D. Ashby, F. M. Toma, J. B. Neaton, I. D. Sharp and A. Weber-Bargioni, Nat. Energy, 2016, 1, 16093.

63 E. M. Miller, Y. Zhao, C. C. Mercado, S. K. Saha, J. M. Luther, K. Zhu, V. Stevanović, C. L. Perkins and J. van de Lagemaat, Phys. Chem. Chem. Phys., 2014, 16, 22122-22130.

64 C. Bi, Q. Wang, Y. Shao, Y. Yuan, Z. Xiao and J. Huang, Nat. Commun., 2015, 6, 7747.
65 Y.-J. Jeon, S. Lee, R. Kang, J.-E. Kim, J.-S. Yeo, S.-H. Lee, S.-S. Kim, J.-M. Yun and D.-Y. Kim, Sci. Rep., 2014, 4, 6953. 66 B. Lee, T. Hwang, S. Lee, B. Shin and B. Park, Sci. Rep., 2019, 9, 4803.

67 X. Zheng, Y. Hou, C. Bao, J. Yin, F. Yuan, Z. Huang, K. Song, J. Liu, J. Troughton, N. Gasparini, C. Zhou, Y. Lin, D.-J. Xue, B. Chen, A. K. Johnston, N. Wei, M. N. Hedhili, M. Wei, A. Y. Alsalloum, P. Maity, B. Turedi, C. Yang, D. Baran, T. D. Anthopoulos, Y. Han, Z.-H. Lu, O. F. Mohammed, F. Gao, E. H. Sargent and O. M. Bakr, Nat. Energy, 2020, 5, 131-140.

68 J. Liu, C. Gao, X. He, Q. Ye, L. Ouyang, D. Zhuang, C. Liao, J. Mei and W. Lau, ACS Appl. Mater. Interfaces, 2015, 7(43), 24008-24015.

69 Q. Liang, J. Liu, Z. Cheng, Y. Li, L. Chen, R. Zhang, J. Zhang and Y. Han, J. Mater. Chem. A, 2016, 4, 223.

70 A. Dualeh, N. Tétreault, T. Moehl, P. Gao, M. K. Nazeeruddin and M. Grätzel, Adv. Funct. Mater., 2014, 24, 3250-3258.

71 L. Huang, Z. Hu, G. Yue, J. Liu, X. Cui, J. Zhang and Y. Zhu, Phys. Chem. Chem. Phys., 2015, 17, 22015. 